\title{
A utilização da Pista Direta de Planas no tratamento precoce da mordida cruzada posterior: relato de caso
}

\author{
The use of Planas Direct Track early treatment of posterior crossbite: \\ a case report
}

\author{
El uso de Pistas Directas Planas para el tratamiento precoz \\ de la mordida cruzada posterior: reporte de un caso
}

\author{
Artênio José Isper GARBIN ${ }^{1}$ \\ Bruno WAKAYAMA ${ }^{2}$ \\ Tânia Adas Saliba ROVIDA ${ }^{1}$ \\ Cléa Adas Saliba GARBIN ${ }^{3}$
}

\begin{abstract}
${ }^{I}$ Professor Adjunto do Programa de Pós-Graduação em Odontologia Preventiva e Social, Faculdade de Odontologia de Araçatuba, UNESP-Univ Estadual Paulista, Araçatuba-SP, Brasil

${ }^{2}$ Mestrando do Programa de Pós-Graduação em Odontologia Preventiva e Social, Faculdade de Odontologia de Araçatuba, UNESP-Univ Estadual Paulista, Araçatuba - SP, Brasil ${ }^{3}$ Professora Titular do Programa de Pós-Graduação em Odontologia Preventiva e Social, Faculdade de Odontologia de Araçatuba, UNESP-Univ Estadual Paulista, Araçatuba - SP, Brasil
\end{abstract}

\begin{abstract}
Resumo
A mordida cruzada posterior é considerada a mais prevalente dentre as oclusopatias, e, se não tratada precocemente pode acarretar em alterações craniofaciais significantes na fase adulta, comprometendo toda a estrutura estomatognática. Objetivou-se relatar dois casos clínicos de mordida cruzada posterior funcional, com a utilização das Pistas Diretas de Planas como tratamento, a fim de determinar sua eficácia e estabilidade oclusal. O $1^{\circ}$ caso clínico refere-se a uma paciente de 6 anos, com mordida cruzada posterior funcional unilateral esquerda e desvio de linha média. Em vista ao protocolo da Reabilitação Neuroclusal e a utilização das Pistas Diretas de Planas como tratramento, foram realizadas as remoções dos contatos prematuros dos dentes 64 e 65. Em seguida foram confeccionadas as Pistas Diretas Planas nos respectivos dentes, em planos inclinado a fim de determinar a correta estimulação neuromuscular e promover o equilibrio funcional do sistema estomatognático. Após 63 dias houve a correção da mordida cruzada e o caso foi acompanhado por 2 anos. O $2^{\circ}$ caso clínico refere-se a uma paciente de 3 anos, com mordida cruzada posterior funcional unilateral direita e desvio de linha média. Seguiu-se o mesmo protocolo de tratamento com a Reabilitação Neuroclusal e confeção das Pistas Diretas de Planas descritos no caso 1, e após 95 dias houve a correção da mordida cruzada posterior e o acompanhamento foi de 2 anos. Conclui-se que as Pistas Diretas de Planas foram eficazes nas correções das mordidas cruzadas posteriores nos dois casos relatados, garantindo a harmonia oclusal e a sua estabilidade após o tratamento.
\end{abstract}

Descritores: Má Oclusão; Ajuste Oclusal; Diagnóstico Precoce.

\begin{abstract}
The posterior crossbite is considered the most prevalent among the malocclusions, can result in significant craniofacial alterations in adulthood, affecting all stomathognatic structure, if not treated early. The aim to report the intervention carried out in two cases of posterior crossbite, with the use of the Planas direct tracks in order to determine their effectiveness and occlusal stability. The $1^{\text {st }}$ case report refers to a female patient, 6 years old was diagnosed with left unilateral posterior crossbite and midline deviation. Given the Neuroclusal Rehabilitation protocol and the use of Planas Direct Tracks as tratramento, there were removals of premature contacts, previously evidenced by carbon paper, the teeth 64 and 65. Then the Planas Direct Tracks were made on their teeth, in planes inclined to determine the correct neuromuscular stimulation and promote the functional balance of the stomatognathic system. After 63 days there was a crossbite correction and monitoring for 2 years to ensure its occlusal stability. The $2^{\text {nd }}$ case report refers to female patient, 3 years old was diagnosed with right unilateral crossbite posterior and midline deviation. This was followed by the same treatment protocol with the Neuroclusal Rehabilitation and confection of Planas Direct Tracks described in case 1 and after 95 days there was a correction of posterior cross bite and the follow-up was 2 years. In conclusion, the Planas Direct Tracks were effective and efficient in the correction of posterior cross bite in the two reported cases, ensuring the occlusal harmony and stability after treatment.
\end{abstract}

Descriptors: Malocclusion; Occlusal Adjustment; Early Diagnosis.

\begin{abstract}
Resumen
La mordida cruzada posterior es considerado a más frecuente entre las maloclusiones, si no es tratada a tiempo, puede dar lugar a alteraciones craneofaciales significativas en la edad adulta, afectando a toda la estructura stomathognatic. Este estudio tiene por objetivo presentar dos casos de mordida cruzada posterior, con el uso de Pistas Directas Planas como el tratamiento con el fin de determinar su eficacia y estabilidad oclusal. El caso clínico $1^{\mathrm{a}}$ se refiere a un paciente de 6 años de edad, con posterior unilateral izquierda mordida cruzada y la desviación de la línea media. En vista de la protocolo de rehabilitación Neuroclusal y el uso de Pistas Directas Planas como tratramento, la eliminación de los contactos prematuros de los dientes 64 y 65 se llevaron a cabo a continuación, las Pistas Directas Planas se hicieron en los dientes, planos inclinados para determinar la estimulación neuromuscular correcta y promover el equilibrio funcional del sistema estomatognático. Después de 63 días hubo una corrección de la mordida cruzada y había un seguimiento durante 2 años. El segundo caso clínico se refiere a un paciente de 3 años de edad, con mordida cruzada posterior derecho unilateral y la desviación de la línea media. Se siguió el mismo protocolo de tratamiento con el Neuroclusal Rehabilitación y confección de Planas directa de pistas que se describen en el caso 1 , y después de 95 días hubo una corrección de la mordida cruzada posterior y el seguimiento fue de 2 años. En conclusión, el directo de pistas Planas fueron eficaces en la corrección de la mordida cruzada posterior en los dos casos reportados, lo que garantiza la armonía y la estabilidad oclusal después del tratamiento.
\end{abstract}

Descriptores: Maloclusión; Ajuste Oclusal ; Diagnóstico Precoz. 


\section{INTRODUÇÃO}

A má oclusão é considerada como um dos principais problemas odontológicos mundiais, devido sua alta dimensão epidemiológica na fase infantil e pelas suas consequências físicas e emocionais na vida adulta ${ }^{1}$. Dessa maneira, a utilização de ações preventivas na primeira infância, a fim de diminuir a incidência das oclusopatias, é a principal forma de enfrentar esse problema de saúde pública ${ }^{2}$.

A mordida cruzada posterior pode ocorrer uni ou bilateralmente, sendo classificada, didaticamente, em esquelética, dentária ou funcional, sendo a última a mais prevalente. Essa alteração oclusal funcional é caracterizada pela existência de contatos prematuros, que depreciam a oclusão, provocando alterações esqueléticas permanentes se não tratada precocemente ${ }^{3}$. Estudos relatam que a permanência da mordida cruzada posterior funcional pode estimular o surgimento de distúrbios temporomandibulares ${ }^{4}$, assimetria facial $^{5}$, depreciação da força de mastigação ${ }^{6}$ e até afetar a qualidade de vida desses indivíduos ${ }^{7}$.

A intervenção precoce dessa oclusopatia é de fundamental importância nessa fase de crescimento, devido os benefícios que lhe serão proporcionados com o correto desenvolvimento crâniofacial ${ }^{8}$. Isto é firmado em decorrência do alto poder de remodelação óssea nesse período, o que influencia na restruturação do sistema estomatognático e na fisiologia crânio-muscular² .

Dada à importância da eleição de um tratamento que atue de forma efetiva e com boa aceitação pelo paciente, requisitos considerados fundamentais para garantir o prognóstico desejado, a Reabilitação Neuroclusal torna-se atrativa para a correção da mordida cruzada posterior, devido aos seus benefícios ortopédicos, bem como por suas vantagens inerentes ao tratamento: facilidade na operação clínica, utilização de recursos simples e de baixo custo e pela colaboração passiva do paciente ao tratamento 9 .

A reabilitação Neuroclusal, firmada por Planas, estabelece em sua filosofia a reprogramação da atividade neuromuscular pela eliminação dos contatos prematuros, a fim de reestruturar o sistema estomatognático ${ }^{10}$. Dessa forma, a excitação neuromuscular proveniente da correta estimulação funcional, com o auxílio das Pistas Diretas de Planas, determinará um padrão de desenvolvimento facial e oclusal harmônico ${ }^{10,11}$.

O tratamento de correção da mordida cruzada posterior utilizando Pistas Diretas de Planas pode ter seu protocolo assim descrito ${ }^{9,11}$ : inicialmente os pontos de contatos prematuros são evidenciados com o auxílio do papel carbono, e em seguida, são realizados os ajustes oclusais necessários. Após a desprogramação estomatognática pela remoção das interferências oclusais, procede-se à confecção das Pistas Diretas de Planas em planos inclinados nos dentes superiores do lado cruzado, utilizando resina composta fotopolimerizável. Para tanto, segue-se o protocolo de uma restauração adesiva, com a profilaxia prévia dos dentes, isolamento do campo, condicionamento ácido com ácido fosfórico $37 \%$ por 30 segundos, aplicação do sistema adesivo, fotopolimerização, inserção de resina composta em planos inclinados $\left(45^{\circ}\right)$, fotopolimerização, ajuste oclusal, acabamento e polimento ${ }^{9,11}$ (Figura1).
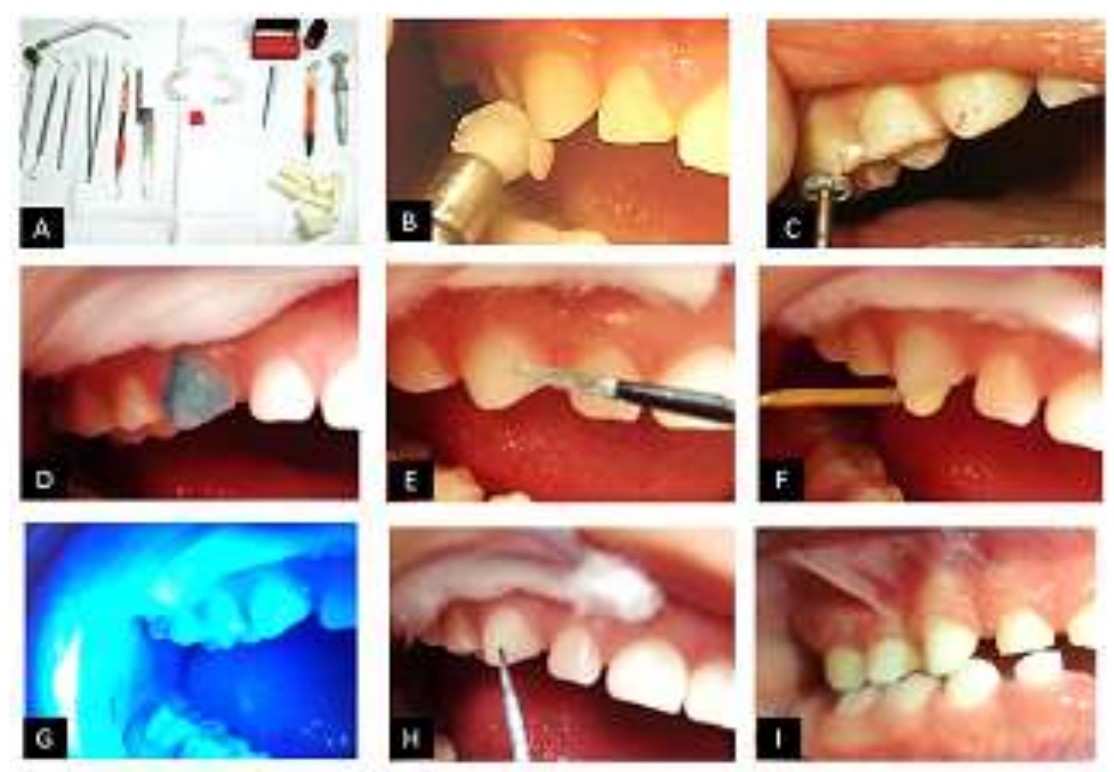

Figura 1. Confecção das Pistas Diretas de Planas ${ }^{9,11}$ A)Mesa Clínica; B) Profilaxia; C) Ajuste Oclusal;

D) Condicionamento Ácido; E) Aplicação do Sistema Adesivo;

F) Inserção da Resina Composta; G) Fotopolimerização;

H) Polimento e Acabamento; I) Pista Confeccionada

Dado o exposto, o presente estudo tem o propósito de relatar dois casos clínicos da intervenção da mordida cruzada posterior funcional, com a utilização das Pistas Diretas de Planas, a fim de determinar sua eficácia e sua estabilidade oclusal.

\section{CASO CLÍNICO 1}

Paciente SBTP, gênero feminino, 6 anos, no exame clinico inicial foi diagnosticada com mordida cruzada posterior funcional unilateral esquerda e desvio de linha média. A paciente não apresentava hábitos de sucção e apresentava trespasse horizontal e vertical normais (Figura 2).
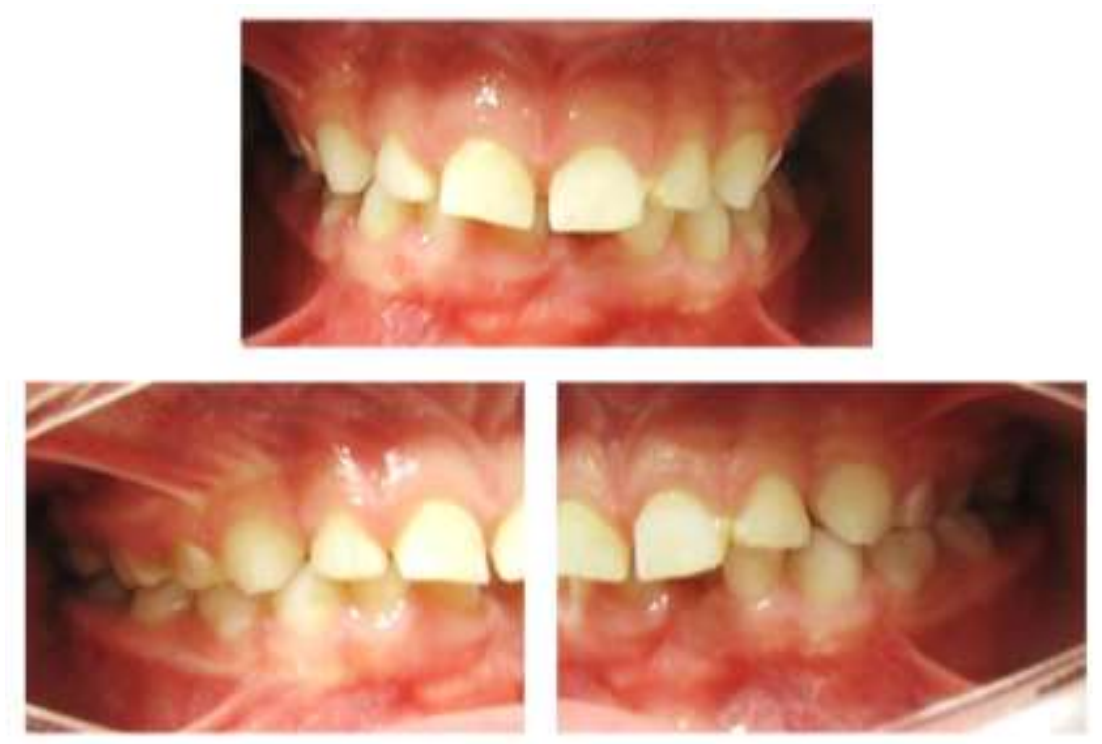

Figura 2. Inicio do tratamento: mordida cruzada posterior unilateral esquerda

Para inicío ao protocolo de tratamento da Reabilitação Neuroclusal, foram realizadas as remoções 
dos contatos prematuros, previamente evidenciadas pelo papel carbono, nos dentes 64 e 65. Em seguida foram confeccionadas as Pistas Diretas Planas nos respectivos dentes, em planos inclinado e posterteiormente realizados o acabamento e o polimento. Após 63 dias houve a correção da mordida cruzada posterior e nivelamento da linha média, o acompanhamento foi de 1 ano (Figura 3) e 2 anos (Figura 4) a fim de verificar a estabilidade oclusal.

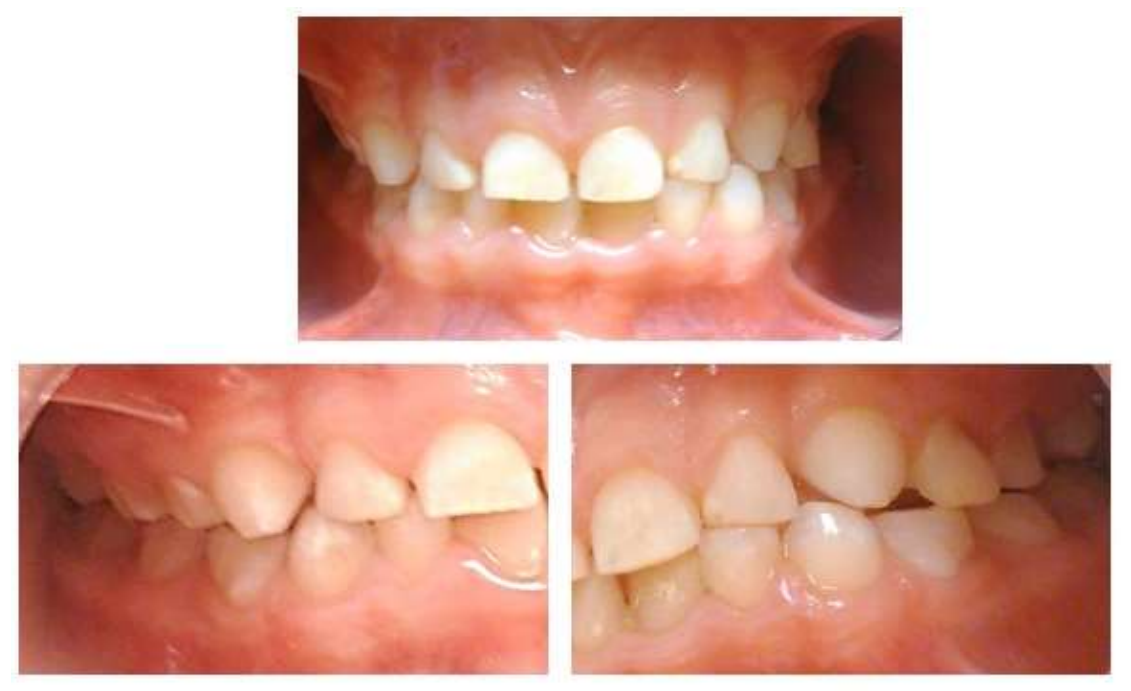

Figura 3. Caso clínico 1: após 1 ano de acompanhamento
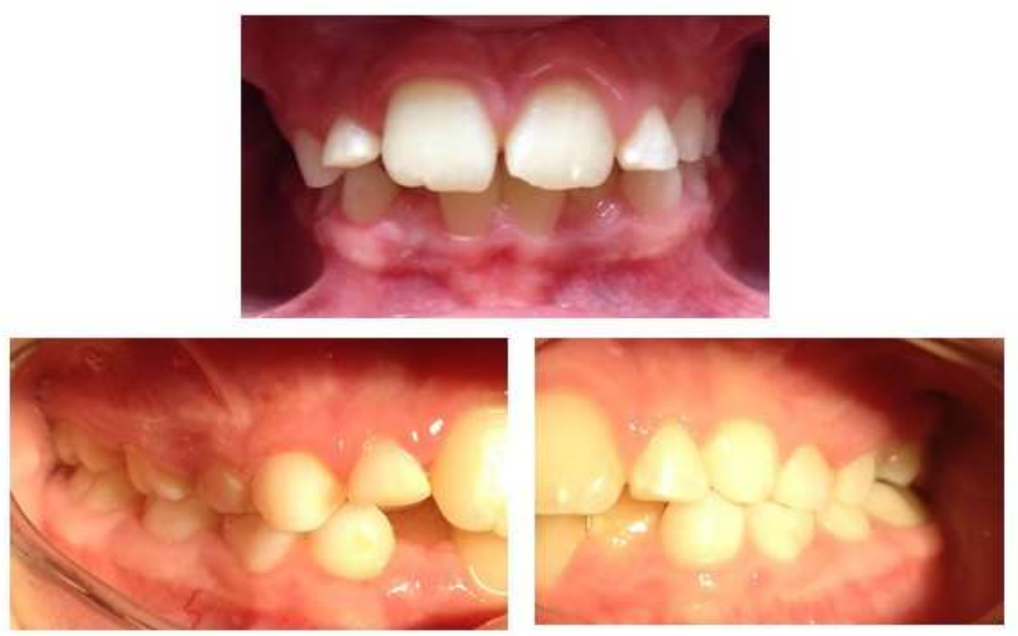

Figura 4. Caso clínico 1: após 2 anos de acompanhamento

\section{CASO CLÍNICO 2}

Paciente VRV, gênero feminino, 3 anos, no exame clinico inicial foi diagnosticada com mordida cruzada posterior funcional unilateral direita, mordida aberta e desvio de linha média. A paciente fazia uso de mamadeira e não foi detectada a presença de apinhamento dental e giroversão (Figura 5).

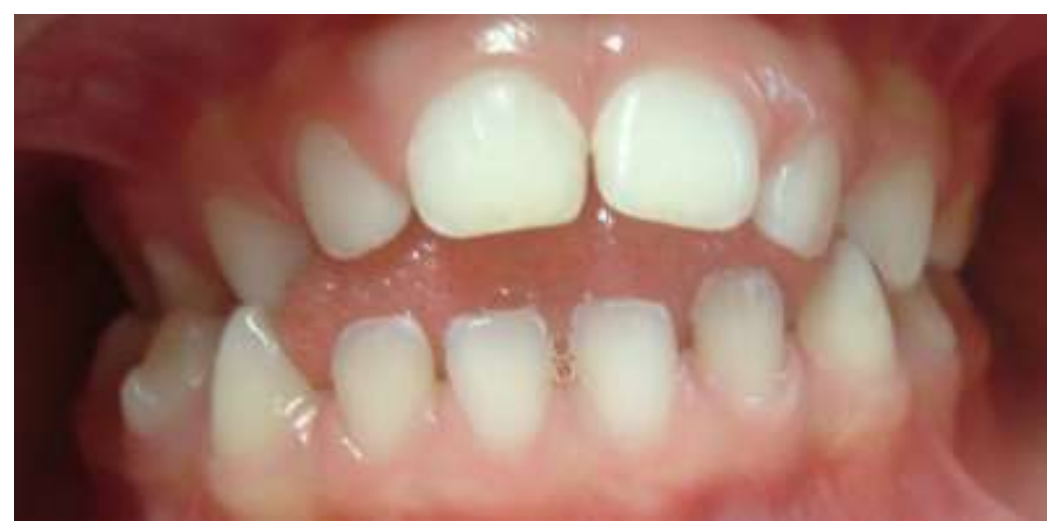

Figura 5. Inicio do tratamento: mordida cruzada posterior unilateral direita

Para o início do tratamento, foram feitas as marcações oclusais com o papel carbono e realizado os desgastes seletivos dos dentes 53, 54 e 55. Após a remoção destes contatos prematuros, confeccionaram-se as Pistas em planos inclinados nos respectivos dentes. Posteriormente, foi realizado o acabamento e o polimento. Após 95 dias houve o restabelecimento oclusal da mordida em questão e o nivelamento da linha média. $\mathrm{O}$ hábito deletério foi cessado e houve o acompanhamento por 2 anos, assegurando a estabilidade oclusal (Figura 6).
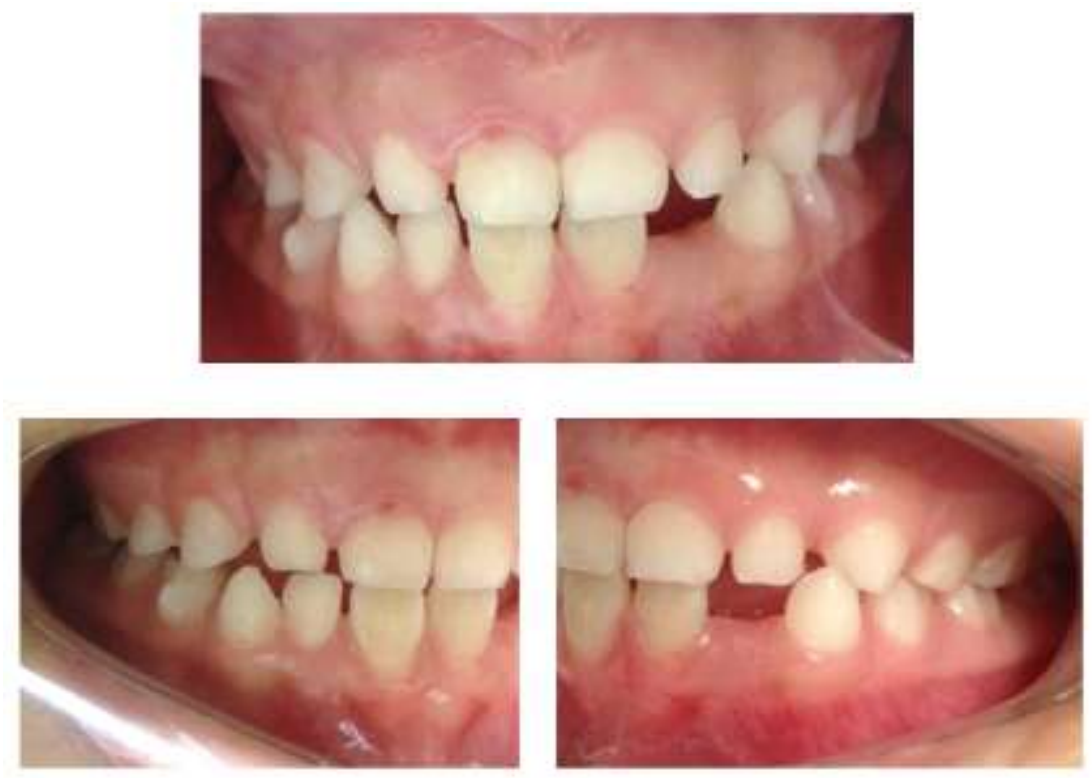

Figura 6. Após 2 anos de acompanhamento

\section{DISCUSSÃO}

Os altos índices de prevalência da mordida cruzada posterior em pré-escolares alertam a necessidade da prevenção e intervenção precoce, visto que essa oclusopatia não se autocorrige e ocasionam em transtornos fisiológicos, sociais e psicológicos nesses indivíduos $^{7,12,13}$.

O tratamento da mordida cruzada posterior na dentição decídua ou mista, apresentam melhores prognósticos devido à possibilidade da atuação da ortopedia funcional nas bases ósseas em crescimento e nas suturas craniofaciais em fusionamento ${ }^{14}$. Entretanto, o tratamento desta oclusopatia na idade adulta, ocasiona a limitação dos recursos a serem utilizados, geralmente necessitando da intervenção cirúrgica para estabelecer o padrão transversal normal ${ }^{15}$.

Nos casos clínicos apresentados, a utilização das Pistas Diretas de Planas, obtiveram-se ótimos resultados em um curto período de tempo de tratamento, como: o nivelamento da linha média, o restabelecimento de um padrão oclusal harmônico e a restruturação da morfologia do sistema estomatognático. Dessa forma, é estabelecido um padrão facial simétrico firmado pela Reabilitação Neuroclusal ${ }^{10}$.

A utilização deste recurso, com os ajustes oclusais e a confecção das Pistas tem sido relatada por autores $^{2,9,11,16,17,18}$ como eficaz e efetivo na correção precoce da mordida cruzada posterior, pela mudança postural mandibular para o correto desempenho funcional estomatognático.

O recurso terapêutico das Pistas Diretas de Planas propiciaram bons prognósticos, devido sua ação em 
determinar uma correta excitação e estimulação nos centros neurais. Isso é estabelecido devido sua ação em reestruturar o padrão de oclusão funcional pelo balanceio da atividade neuromuscular refletindo no bom desenvolvimento das estruturas crânio musculares ${ }^{10,18,19}$. Visto isto, é de grande relevância a disseminação dessa técnica devido às vantagens implícitas ao tratamento, bem como pela possibilidade de sua execução nos consultórios privados e públicos por clínicos gerais.

\section{CONCLUSÃO}

Conclui-se que, as Pistas Diretas de Planas foram eficazes nas correções das mordidas cruzadas posteriores assegurando sua estabilidade oclusal.

\section{REFERÊNCIAS}

1. Primozic J, Richmond $\mathrm{S}, \mathrm{Kau} \mathrm{CH}$, Zhurov A, Ovsenik M .Three-dimensional evaluation of early crossbite correction: a longitudinal study. Eur J Orthod. 2013 Feb; 35(1):7-13.

2. Garbin AJI, Wakayama B, Santos RR, Rovida TAS, Garbin CAS. Pistas Diretas Planas para o tratamento de mordida cruzada posterior. Rev Cubana Estomatol. 2014; 51(1):113-20.

3. Locks A, Weissheimer A, Ritter DE, Ribeiro GLU, Menezes LM, Carla D’Agostini Derech CD, et al. Mordida cruzada posterior: uma classificação mais didática. R Dental Press Ortodon Ortop Facial. 2008 Mar/Abr; 13(2):146-158.

4. Thilander B, Bjerklin K. Posterior crossbite and temporomandibular disorders (TMDs): need for orthodontic treatment? Eur J Orthod. 2012 Dec; 34(6):667-73.

5. Kwak YY, Jang I, Cho DS, Chai BK. Functional evaluation of orthopedic and orthodontic treatment in a patient with unilateral posterior crossbite and facial asymmetry. Coreano J Orthod. 2014 May; 44(3):143-53.

6. Primozic J, Richmond $\mathrm{S}, \mathrm{Kau} \mathrm{CH}$, Zhurov A, Ovsenik M.Three-dimensional evaluation of early crossbite correction: a longitudinal study. Eur J Orthod. 2013 Feb; 35(1):7-13.

7. Rosa GN, Del Fabro JP, Tomazoni F, Tuchtenhagen S, Alves LS, Ardenghi TM. Association of malocclusion, happiness, and oral healthrelated quality of life (OHRQoL) in schoolchildren. J Public Health Dent. 2015 Jul. doi: 10.1111/jphd.12111.

8. Proffit WR. The timing of early treatment: an overview. Am J Orthod Dentofacial Orthop. 2006 Apr; 129(4):47-9.

9. Chibinski ACR, Czlusniak GD, Melo MD. Pistas diretas planas: terapia ortopédica para correção de mordida cruzada funcional. R Clin Ortodon Dental Press. 2005; 4(3):64-72.
10. Planas P. Reabilitação Neuroclusal. $2^{a}$ ed. Rio de Janeiro: Medsi;1997.

11. Garbin AJI, Wakayama B, Rovida TAS, Garbin CAS. Reabilitação neuroclusal como tratamento da mordida cruzada posterior: relato de caso. Braz J Surg Clin Res. 2015 Jul; 11(4):21-24.

12. Oliveira CM, Sheiham A. Orthodontic treatment and its impact on oral health-related quality of life in brazilian adolescents. J Orthod. 2004 mar; 31(1):207.

13. Dimberg L, Arnrup K, Bondemark L. The impact of malocclusion on the quality of life among children and adolescents: a systematic review of quantitative studies. Eur J Orthod. 2015 Jun; 37(3):238-247.

14. Simões W. Ortopedia Funcional dos Maxilares Através da Reabilitação Neuro-Oclusal. $3^{\circ}$ ed. Artes médicas; 2003.

15. Albuquerque Neto AD, Sampaio TRC, SantoS DLP, Nogueira Filho LLT, Laureano Filho JR, Nogueira PTBC. Expansão rápida maxilar cirurgicamente assistida com o uso de distrator ósseo-ancorado: relato de caso. Braz J Surg Clin Res. 2015; 11(1):247.

16. Gribel MN. Planas Direct Tracks in the early treatment of unilateral crossbite with mandibular postural deviation: Why worry so soon? World J Orthod. 2002;3:239-49.

17. Ramirez-Yañez GO. Planas direct tracks for early crossbite correction. J Clin Orthod. 2003 jun;37(6):294-8.

18. Chibinski ACR, Czlusniak GD. Evaluation of treatment for functional posterior crossbite ofthe deciduous dentition using Planas' direct tracks. Indian J Dent Res. 2011; 22(5):654-58.

19. Castro LO, Valladares-Neto J, Estrela C. Prevalência de Maloclusão em Indivíduos que Solicitaram Tratamento Ortodôntico na Rede Pública de Saúde. Rev Odontol Bras Central. 2010;19(51):223-326.

\section{CONFLITO DE INTERESSES}

Os autores declaram não haver conflitos de interesse.

\section{AUTOR PARA CORRESPONDÊNCIA}

Tânia Adas Saliba Rovida

tasalibarovida@foa.unesp.br

Submetido em 15/05/2016 Aceito em 27/06/2016 